\title{
DISCOVERY OF A PLANETARY NEBULA ASSOCIATED WITH THE WHITE DWARF GD 561
}

\author{
R. NAPIWOTZKI and D. SCHÖNBERNER \\ Institut für theoretische Physik und Sternwarte der Universität, Olshausenstr. 40, \\ W-2300 Kiel, Germany
}

The search for old planetary nebulae around hot white dwarfs, PG 1159 stars, and sdO stars is subject of intensive studies (e.g. Kwitter et al. 1989). We report here on the discovery of such a nebula around the white dwarf GD 561 .

GD 561 (WD 2342+806) was discovered by Giclas et al. (1970). It was classified as DAO by Bergeron et al. 1992. A comparison of a spectrogram of GD 561, taken by us, with the DAO central stars of S 216 and NGC 7293 shows striking similarity. From a preliminary analysis of the Balmer lines we got $T_{\text {eff }}=50,000 \mathrm{~K} \ldots 60,000 \mathrm{~K}$. But according to the discussion in Napiwotzki (1992 and these proceedings) this determination needs further scrutiny.

In the background of our exposure the forbidden $O$ III lines at $4959 \AA$ and $5007 \AA$, typical for a PN, are clearly visible. The associated nebula was listed before in the catalogue of Sharpless (1959) as H II region (S 174). The distance of S 174 was determined by Fich \& Blitz (1984) from its radial velocity using the galactic rotation curve to be $220 \mathrm{pc}$. The distance is in agreement with the estimated distance of GD 561. The resulting radius $(0.3 \mathrm{pc})$ is consistent with the interpretation of $\mathrm{S} 174$ as beeing an old PN. The position of GD 561 is not at the center but near the outer rim of the PN. But this is not unexpected: the central stars of very old PN are very often off-center. We conclude that GD 561 is the central star of S 174 .

\section{References}

Bergeron, P., Saffer, R.A., Liebert, J., 1992, ApJ, in press

Fich M., Blitz, L., 1984, ApJ 279, 125

Giclas, H.L., Burnham, R., Thomas, N.G., 1970, Lowell Obs. Bull., 7, 183

Kwitter, K.B., Massey, P., Congdon, C.W., Pasachoff, J.M., 1989, ApJ 97, 1423

Napiwotzki, R., 1992, Proc. Kiel/CCP7 workshop on Atmospheres of Early Type

Stars, eds. U. Heber, S. Jefferey, Springer, p. 310

Sharpless, S., 1959, ApJS 4, 257 